\title{
Entwicklung eines Verfahrens und einer Anlage zum Sortieren von Altkorken
}

\author{
DIPL.-ING. FRANK SCHÖNUNG \\ DiPL.-ING. MARTIN MITTWOLLEN \\ PROF. DR.-ING. KAI FURMANS \\ INSTITUT FÜR FÖRDERTECHNIK UND LOGISTIKSYSTEME (IFL), \\ UNIVERSITÄT KARLSRUHE (TH)
}

\section{Zusammenfassung}

Der Naturwerkstoff Kork wird dank seiner elastischen Eigenschaft und seines guten Wärmeisolationsverhaltens in Industrieprodukten, in der Baustofftechnologie, als Flaschenverschlüsse und in Freizeitprodukten eingesetzt. Aufgrund des Preises und der Verfügbarkeit des Werkstoffs werden Korkprodukte heute wiederverwertet. Den größten Anteil der Altkorken stellen dabei die Flaschenverschlüsse dar. Da sich in den letzten Jahren Kunststoffweinstopfen aufgrund ihrer konstanten Qualität immer größerer Beliebtheit erfreuen, sind diese auch immer häufiger an den Sammelstellen für Altkorken wieder zu finden. Eine manuelle Trennung der Kunststoffflaschenstopfen von den Naturkorken vor der Wiederverwertung ist sehr zeitaufwändig, weshalb die Forderung entstand diesen Prozess zu automatisieren. Da keine entsprechenden Maschinen verfügbar sind, musste ein neues Verfahren und eine Anlage zum Erfüllen dieser Aufgabe entwickelt werden. Dieser Beitrag stellt die wichtigsten Schritte von den wissenschaftlichen Untersuchungen bis zur Entstehung des Prototyps einer Korksortieranlage vor.

\begin{abstract}
Cork is, due to its elastic and heat insulating properties, used in industry, construction, as bottle caps and in leisure products. Price and availability of the material are the motive for cork recycling, in particular of cork bottle caps. As plastic bottle corks provide a constant and high quality, their amount at the cork collecting points has increased during the last years. As a manual separation of plastic and natural corks is very time consuming and exhausting, there is a demand on the automation of the sorting process. To meet the demands a new process and machine had to be developed. This essay presents the most important steps beginning at the research up to the realisation of a prototype.
\end{abstract}

\section{Einleitung}

Kork gehört zu den Naturwerkstoffen, die innerhalb eines Recycling-Prozesses wiederverwertet werden können. Kork-Recyclingprodukte sind beispielsweise Pinwände, Bodenbeläge und Dämmmaterialien - alles Produkte, die aus Korkgranulat hergestellt werden. In Deutschland werden Altkorken an dafür eingerichteten Korksammelstellen gesammelt. Das Material wird in einer zentralen Sortiereinrichtung von Fremdstoffen befreit, auf seine Qualität geprüft und anschließend als wertvoller Recycling-Naturwerkstoff für die Produktion von Korkprodukten zur Verfügung gestellt. Für die Wiederverwertung geeignet sind Naturkorken, Presskorken und Feingranulatkorken, die als Weinverschlüsse, Sektverschlüsse, in Form von Griffkorken, als konische Flaschenverschlüsse, als Korkplatten und in anderen Formen auftreten. Fremdstoffe müssen aus dem Recyclingkreislauf entfernt werden. Dazu gehören Altbatterien, Griffkorken mit Kunststoff- oder Holzgriff, stark verschmutzte Korken, Papier, Kunststoffteile, Metallteile, sonstiger Müll und in zunehmendem Maße Kunststoffflaschenstopfen. Das Aussortieren dieser Stoffe erfolgte bisher manuell. 


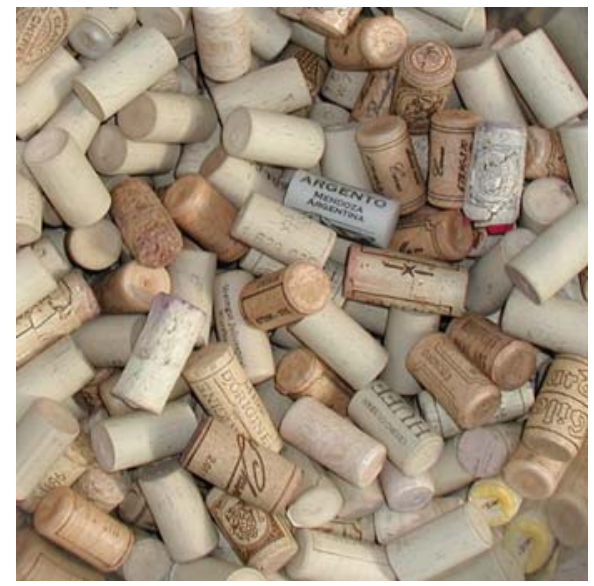

Abbildung 1: verschiedene Natur- und Kunststoffflaschenstopfen

\section{Problemstellung}

Beim manuellen Aussortieren der Fremdstoffe ist insbesondere das Erkennen der Kunststoffflaschenstopfen aufgrund der zum Teil großen optischen Ähnlichkeit mit den Naturkorken eine zeitaufwändige Arbeit, die mit Zunahme des Kunststoffkorkanteils die Gesamtsortierleistung der Korksortiereinrichtung immer weiter reduziert hat. Um die Sortierleistung zu steigern, entstand deshalb die Forderung diesen Teilprozess zu automatisieren. Dabei müssen folgende Anforderungen erfüllt werden: Die Korken dürfen für den Sortierprozess nicht zerkleinert werden, damit die Korngröße nach der Sortierung individuell dem Recyclingprodukt angepasst werden kann. Die Einrichtung muss eine Leistung von $4000 \mathrm{~m}^{3} / \mathrm{a}$ erreichen. Das entspricht einem Durchsatz von etwa $\lambda=40$ Korken/s. Der Anteil der Kunststoffflaschenstopfen beträgt im Jahre $2005<5 \%$ und wird in den kommenden Jahren voraussichtlich auf über 20\% ansteigen. Alle weiteren Fremdstoffe unterscheiden sich von den „Gutkorken“ entweder in ihrer Geometrie oder ihrer Farbgebung deutlich und können deshalb weiterhin vorab manuell aussortiert werden. Zum Abscheiden von FE-Metallen sollte zusätzlich ein Magnetscheider eingesetzt werden, da beispielsweise abgebrochene Korkenzieher, die im Korken verbleiben, optisch nicht zu erkennen sind und nachfolgende Bearbeitungsmaschinen schädigen können.

\section{Vorgehensweise}

Nach Klärung der Anforderungen an eine Korksortiereinrichtung mit der oben beschriebenen Funktionalität wurde überprüft, ob sich herkömmliche Sortieranlagen für diese spezielle Anwendung adaptieren lassen. Um dies zu beurteilen waren die Eigenschaften der Korken nicht ausreichend bekannt, bzw. die Verfahren bedingt durch die aufgeführten Restriktionen nicht geeignet. Exemplarisch kann hier die elektrostatische Sortierung genannt werden, die sich ausschließlich für Sortiergut mit Korngrößen bis etwa $5 \mathrm{~mm}$ eignet. Für das weitere Vorgehen war es deshalb erforderlich, die Eigenschaften der unterschiedlichen Korktypen zu kennen. Dabei reicht es nicht aus die Werkstoffeigenschaften von Naturkork und der eingesetzten Kunststoffe zu kennen, vielmehr müssen die Eigenschaften der Ganzkorken, wie sie bei der Sortierung vorliegen, ermittelt werden. Somit wird sichergestellt, dass Beschichtungen, typische Verschmutzungen und typische Beschädigungen der Altkorken berücksichtigt werden. Aus den ermittelten Daten wurden Trennkriterien bestimmt, die die Unterscheidung von Natur-, Press- und Feingranulatkorken einerseits und Kunststoffflaschenstopfen andererseits ermöglicht. Für die einzelnen Trennkriterien wurde wiederum überprüft, ob sich eine herkömmliche Sortieranlage mit einfachen Mitteln für die Trennung der Korken anpassen lässt. Da für keines der Kriterien eine einfache Anlagenanpassung ausreichend war, mussten eigene Sortierkonzepte entwickelt werden. Für alle entwickelten Sortierkonzepte wurde eine Einschränkung des Sortierguts auf Wein-, Sekt-/Champagner-, Naturkork-Griffkorken und konische Korken bis $\varnothing 35 \mathrm{~mm}$ und einer Länge von 35mm, vorgenommen. Korkplatten und sonstige Korken $>35 \mathrm{~mm}$ sind daher bei der manuellen Vorsortierung mit zu entfernen. Weiterhin ist es erforderlich die Korken, die nach der Vorsortierung über einen Gurtbandförderer zur Sortieranlage gefördert werden, zu vereinzeln und für fast alle Konzepte, die Korken auszurichten. Zur Lösung dieser Teilfunktionen waren weitere empirische Untersuchungen erforderlich, da keine detaillierten Schüttgutmodelle für Schüttgüter mit derart unterschiedlichen Eigenschaften verfügbar sind. 


\section{Das Sortiergut}

Das Sortiergut umfasst mit der oben genannten Einschränkung vier grundsätzliche Korkbauarten: Weinkorken, Sekt- und Champagnerkorken, Griffkorken und konische Korken. Jede der Bauarten ist mit mehreren unterschiedlichen Maßen vertreten (Tabelle 1). Über 50 unterschiedliche Kunststoffflaschenstopfen wurden bisher untersucht. Für die Bestimmung eines oder mehrerer Trennkriterien mussten sowohl die geometrischen als auch die physikalischen Eigenschaften der Korken ermittelt werden. Bisherige Untersuchungen beziehen sich in vielen Fällen auf für den Weinbau erforderliche Daten. Veröffentlichte Untersuchungen der Forschungsanstalt für Garten- und Weinbau in Geisenheim etwa machen Angaben über Kräfte im Korkschloss für unterschiedliche Korkwerkstoffe, die zwar ein Maß für die Steifigkeit der Korken sind, für eine Beurteilung als Trennkriterium jedoch untauglich sind. Um aussagekräftige Daten der physikalischen Eigenschaften von Korken zu erhalten, waren deshalb verschiedenste Messungen unabdingbar.

$\mathrm{Zu}$ den durchgeführten Untersuchungen gehören Spektroskopien in den Wellenlängenbereichen UV, VIS, NIR, MIR, Zug-/Druckversuche zur Bestimmung von Steifigkeit und Dämpfungsverhalten, die Untersuchung thermischer Eigenschaften und die Untersuchung elektrischer, bzw. triboelektrischer Eigenschaften.

Tabelle 1 :Herstellung und Aufbau der Korken

\begin{tabular}{|c|c|c|c|}
\hline & Naturkork & $\begin{array}{l}\text { Press-/Fein- } \\
\text { Granulat-Kork }\end{array}$ & Kunststoffkork \\
\hline Werkstoffe & Naturkork & $\begin{array}{l}\text { Naturkorkgranulat, } \\
\text { Bindemittel }\end{array}$ & $\begin{array}{l}\text { Thermoplaste, PE, PP, EVA } \\
\text { (Ethylen Vinyl Acetat mit } \\
\text { extrudierter Außenhaut aus } \\
\text { SBS Elastomer, Fa. Volpini) }\end{array}$ \\
\hline $\begin{array}{l}\text { Herstellung/ } \\
\text { Behandlung }\end{array}$ & $\begin{array}{l}\text { In Chlorbad gebleicht, } \\
\text { zur Bekämpfung von } \\
\text { Korkbakterien auch } \\
\text { anderen chemischen } \\
\text { Behandlungsmethoden } \\
\text { unterworfen, evtl. noch } \\
\text { Bestrahlung, evtl. } \\
\text { Überzug mit } \\
\text { Gleitschicht, z.B. } \\
\text { Silikon }\end{array}$ & $\begin{array}{l}\text { Beim Verpressen } \\
\text { werden je nach } \\
\text { Bindemittel etwa } 8 \\
\text { Gewichtsprozent } \\
\text { der Korkmasse an } \\
\text { Klebstoff } \\
\text { hinzugegeben. }\end{array}$ & $\begin{array}{l}\text { Spritzgießverfahren } \\
\text { Ein Werkstoff, geschäumt, } \\
\text { Randbereiche sehr feinporig } \\
\text { (Schaum fällt an gekühltem } \\
\text { Werkzeug zusammen) } \\
\text { Extrusionsverfahren } \\
\text { Schlauchhülle, geschäumter } \\
\text { Kern } \\
\text { (Koextrusion) }\end{array}$ \\
\hline Maße (typisch) & $\begin{array}{l}\varnothing 23 \times 33, \varnothing 23 \times 39, \\
\varnothing 23 \times 44, \varnothing 24 \times 33, \\
\varnothing 24 \times 38, \varnothing 24 \times 45, \\
\varnothing 24 \times 49, \varnothing 24 \times 54\end{array}$ & $\begin{array}{l}\varnothing 22,5 \times 33 \\
\varnothing 22,5 \times 36 \\
\varnothing 22,5 \times 38 \\
\varnothing 22,5 \times 42\end{array}$ & 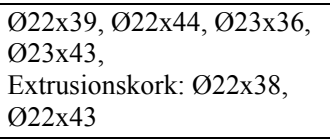 \\
\hline Aussehen & Bedruckt, unbedruckt & $\begin{array}{l}\text { Bedruckt, } \\
\text { unbedruckt }\end{array}$ & $\begin{array}{l}\text { Bedruckt, unbedruckt, } \\
\text { eingefärbt, korkstrukturiert, } \\
\text { leicht strukturiert, } \\
\text { aufgeprägte Schrift/Symbole } \\
\text { an Stirnseiten }\end{array}$ \\
\hline
\end{tabular}

Exemplarisch werden im Folgenden drei Untersuchungen vorgestellt. Dabei sind die folgenden Proben für KorkRecyclingprodukte geeignet: Probe $1=$ Weinnaturkork, Probe $2=$ Weinpresskork, Probe $8=$ Weinfeingranulatkork, Probe 14 = Sekt-/Champagnerkork. Bei den übrigen Korken handelt es sich um Kunststoffflaschenstopfen.

\subsection{Mechanische Eigenschaften: Druckversuch}

Zur Bestimmung des Dämpfungsverhaltens und der Steifigkeit der Korken wurden auf einer Zug-DruckPrüfmaschine die auftretenden Kräfte beim Zusammendrücken der Korken über dem Weg aufgezeichnet. Da für die Abdichtung der Weinverschlüsse das elastische Verhalten der Naturkorken genutzt wird, um eine möglichst gleichmäßige Flächenpressung zwischen Stopfen und Flasche zu erzielen, war zu erwarten, dass gerade das elastische Verhalten der Kunststoffflaschenstopfen dem der Naturkorken ähnlich ist.

Diese Vermutung wurde bei den Messungen auf der Zug-Druck-Prüfmaschine bestätigt. Auch das Dämpfungsverhalten ist aufgrund der Gaseinschlüsse bei beiden Korkarten sehr ähnlich. 


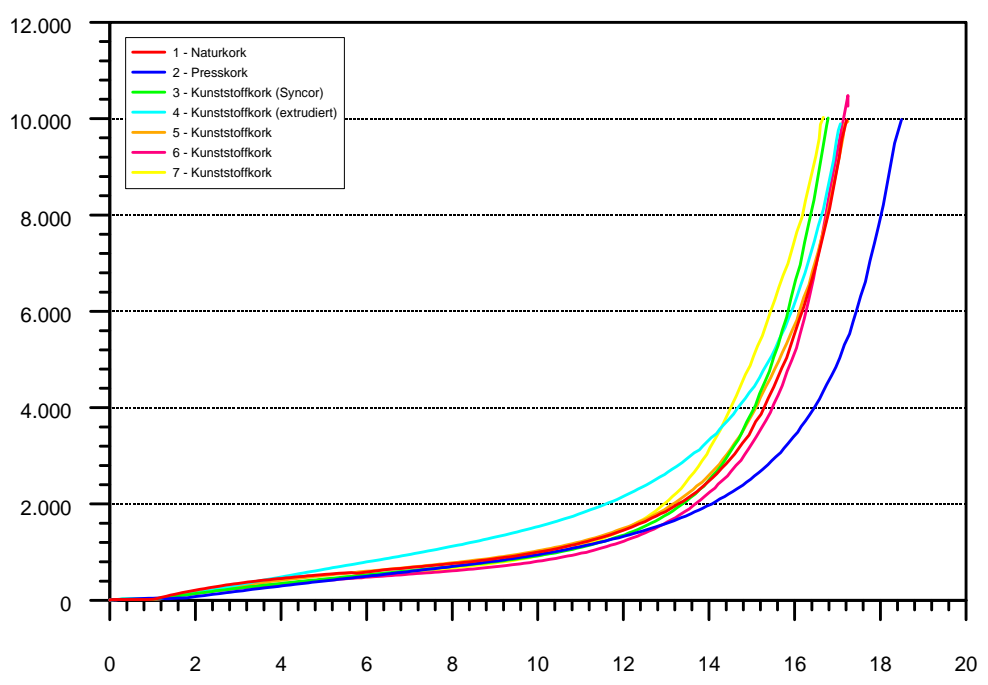

Abbildung 2: Kraft-Weg-Verflauf beim Druckversuch

\subsection{Optische Eigenschaften: Reflexions- und Fluoreszenzmessung}

Im Bereich des UV-Lichts wurden die Korkproben mit monochromatischem Licht der Wellenlängen 200...500nm bestrahlt und die Intensität von Reflexion bzw. Fluoreszenz über einen weiteren Monochromator + Sensor in den Wellenlängen 250..500nm (Abbildung 3) gemessen. Vorteil dieses Verfahrens im Gegensatz zur gewöhnlichen Spektroskopie ist, dass hier auch Fluoreszenzeigenschaften der Korkproben gemessen werden können.

Am Schaubild (Abbildung 3) ist exemplarisch zu erkennen, dass die Natur-, Press-, und Feingranulatkorken (Proben 1, 2, 8 und 14) über ein breites Spektrum Fluoreszenz aufweisen, während bei den Kunststoffflaschenstopfen lediglich bei sehr kurzen Wellenlängen $(200 . .300 \mathrm{~nm})$ Fluoreszenz gemessen wurde. Die Reflexion in diesem Wellenlängenbereich ist dagegen bei den Kunststoffflaschenstopfen deutlich stärker.

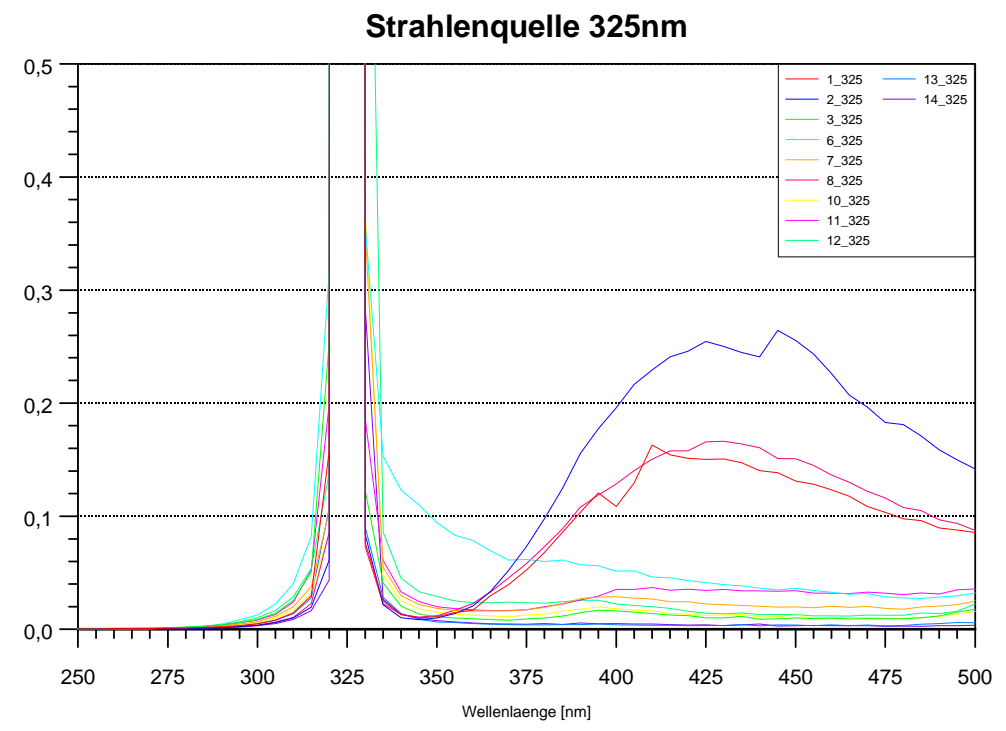

Abbildung 3: Reflexion und Fluoreszenz bei Bestrahlung mit 325nm

Bei einer breitbandigen Bestrahlung im UV-Bereich (Abbildung 4) konnte das gleiche Verhalten gezeigt werden. Nachteilig sind die großen Intensitätsunterschiede zwischen dem reflektierten und dem fluoreszierten Anteil. 


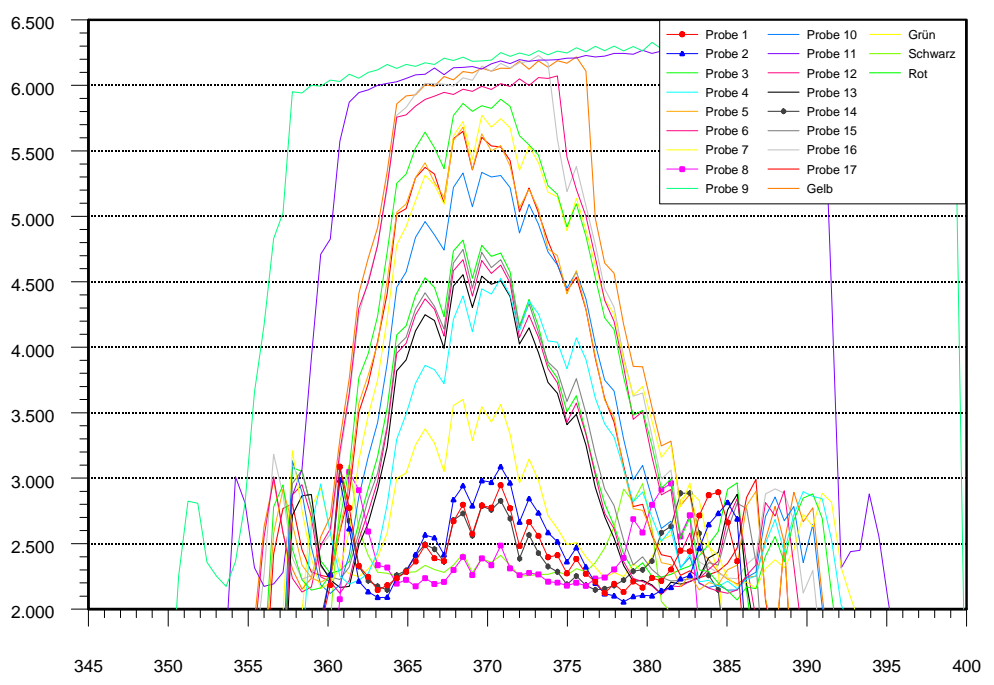

Abbildung 4: Reflexion bei breitbandiger Bestrahlung im UV-Bereich

Bei einer breitbandigen Bestrahlung im NIR-Bereich (Abbildung 5) sind über die Reflexion nur wenige Korken eindeutig der Natur- oder der Kunststofffraktion zuzuordnen.

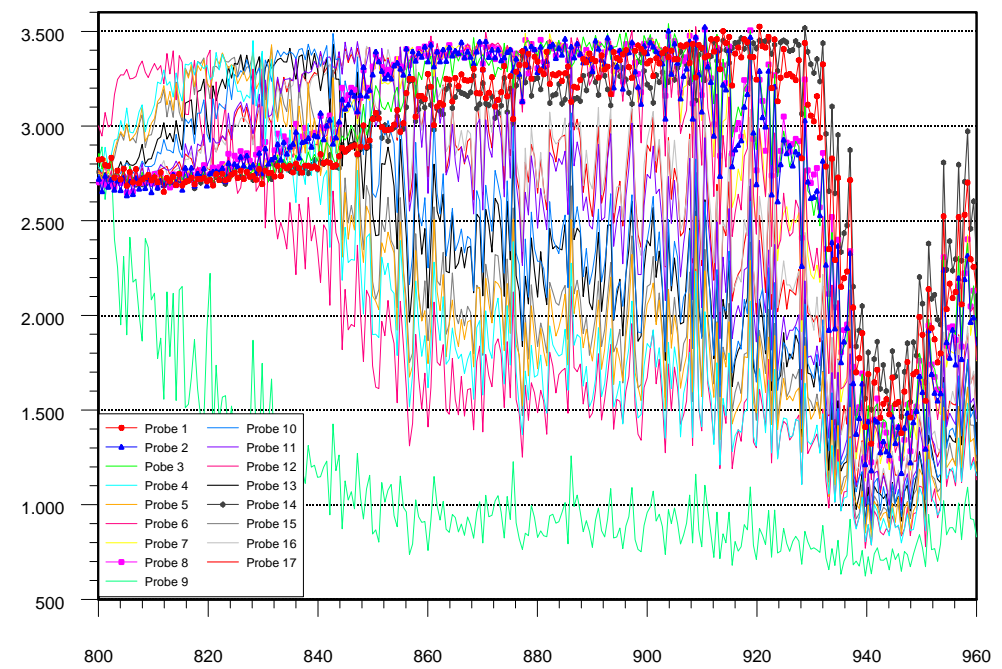

Abbildung 5: Reflexion bei breitbandiger Bestrahlung im NIR-Bereich

\subsection{Optische Eigenschaften: Farbanteile bei Bestrahlung mit Schwarzlicht}

Aufgrund der Ergebnisse der UV-Spektroskopie wurde vermutet, dass auch bei Bestrahlung mit Schwarzlicht deutliche Unterschiede im abgegebenen Spektrum auftreten. Zunächst wurden die Korken zur Sichtprüfung mit Schwarzlicht bestrahlt. Als Schwarzlichtquellen kamen Schwarzlicht-Leuchtstoffröhren und HQV-Lampen zum Einsatz. Das Spektrum der Schwarzlichtröhre beschränkt sich auf UV und blau, während das Spektrum der HQV-Lampe zusätzlich einen starken Rotanteil aufweist.

Abbildung 6 zeigt das volle Reflexionsspektrum im sichtbaren VIS-Bereich bei Bestrahlung mit Schwarzlicht, aufgenommen mit einer CCD-Kamera. Es ist deutlich $\mathrm{zu}$ erkennen, dass im Allgemeinen die Kunststoffflaschenstopfen sehr stark im Blauen reflektieren, während die Naturkorken und drei der Kunststoffflaschenstopfen weiß abstrahlen. Durch Trennen der Farbkanäle (RGB-Farbformat) (Abbildung 7 bis Abbildung 9) ist unter Laborbedingungen eine Unterscheidung im Grünanteil möglich (Abbildung 8). 


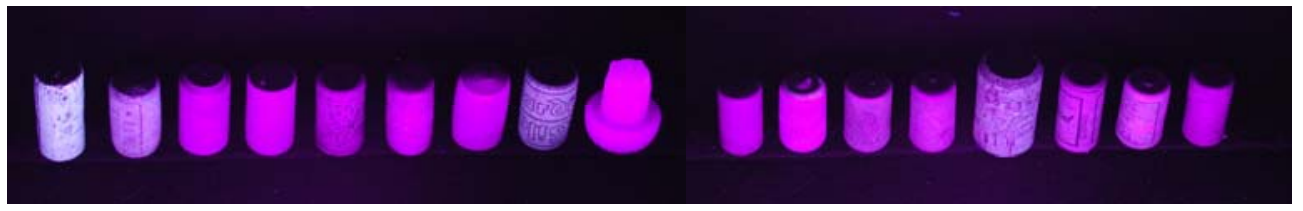

Abbildung 6: RGB bei Bestrahlung mit Schwarzlichtquelle

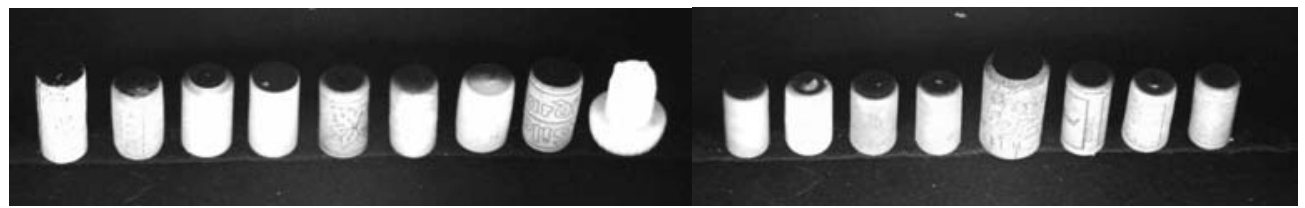

Abbildung 7: Blauanteil bei Bestrahlung mit Schwarzlichtquelle

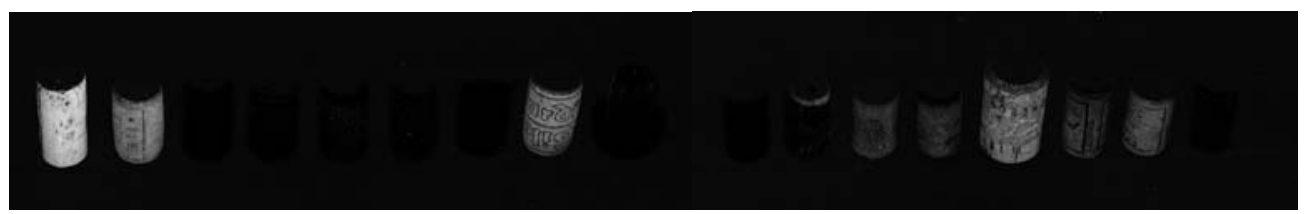

Abbildung 8: Grünanteil bei Bestrahlung mit Schwarzlichtquelle

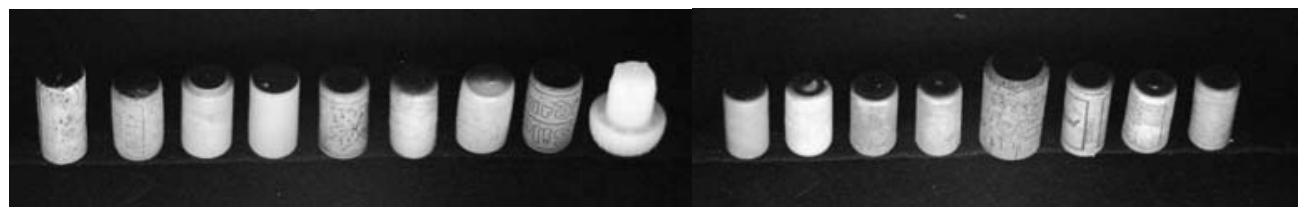

Abbildung 9: Rotanteil bei Bestrahlung mit Schwarzlichtquelle

\subsection{Anregung mit Mikrowellen}

Das Verhalten der Korken bei Anregung durch Mikrowellenbestrahlung ist in erster Linie vom Wassergehalt der Proben abhängig. Zur Bestimmung wurden die Korken der Bestrahlung einer Mikrowellenstrahlenquelle ausgesetzt. Die Temperatur der Korken wurde mit einem Infrarot-Thermometer vor und nach der Bestrahlung gemessen. Die Messergebnisse sind im nachfolgenden Diagramm dargestellt.

Es ist zu erkennen (Abbildung 10), dass Natur-, Press- und Feingranulatkorken sich stark erhitzen, während die Kunststoffflaschenstopfen fast keine Temperaturerhöhung aufweisen. Großen Einfluss auf dieses Verfahren hat die Feuchtigkeit der Korkoberfläche. Dieser kann durch eine größere Bestrahlungsdauer minimiert werden (Wasser an der Oberfläche verdampft). Problematisch bei längeren Bestrahlungsdauern ist allerdings, dass Natur-, Press- und Feingranulatkorken durch die gleichmäßige Erwärmung sehr leicht aufquellen.

Ein alternatives Verfahren stellt die Mikrowellenfeuchtemessung dar. Derzeit finden weitere Untersuchungen mit einem an die Prozessbedingungen angepassten Mikrowellenfeuchtemessverfahren statt. 


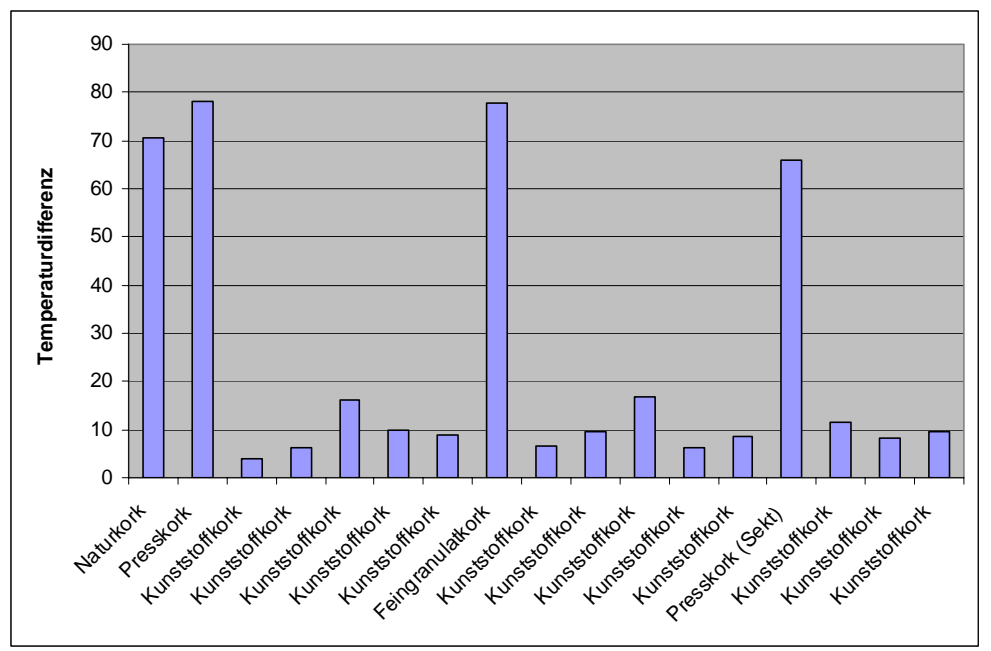

Abbildung 10: Temperaturdifferenz bei Mikrowellenbestrahlung

\section{Vereinzelung und Ausrichtung}

Wie zuvor beschrieben sind für alle entwickelten Sortierkonzepte die Korken zu vereinzeln oder sogar auszurichten. Die größte Schwierigkeit stellen dabei die Unterschiede in Form und Größe der Korken dar. Die Durchmesser reichen von 20mm (Weinkork) bis zu Durchmessern von 32mm (Sektkork) und 35mm (konische Korken). Die Längen sind ebenfalls unterschiedlich. Einrichtungen wie Wendelschwingförderer mit Kulissen wie sie in der Montage für die Vereinzelung und Ausrichtung von Kleinteilen (z.B. Muttern) zum Einsatz kommen, scheiden für die Vereinzelung aus, da sie sich identische Form und Größe der Objekte zu Nutze machen.

Da für die Beschreibung des Schüttgutverhaltens von Korkgemischen keine Modelle verfügbar sind, müssen unterschiedliche Vereinzelungstechniken sowie das Schüttgutverhalten des Korkgemischs deshalb empirisch untersucht werden.

Die aus verschiedenen Ansätzen gewählte Lösung des Vereinzelungsproblems, die auch im Prototyp umgesetzt wurde, wird im Folgenden kurz erläutert. Zur Veranschaulichung dient Abbildung 11. Die Korken werden aus einem Bunker über eine mitnehmerbestückte Walze (1) gefördert und treffen bei (2) auf die im V angeordneten Blechprofile und die Rundriemen der Kanäle. Der Durchmesser der Walze und die Anzahl der Mitnehmer sind für die Gleichmäßigkeit der Förderung verantwortlich. Die Korken rutschen in das Zentrum des Kanals auf zwei sich in Förderrichtung bewegende Riemen. Durch die Kombination der ortsfesten Profile und der sich bewegenden Riemen werden die Korken der Länge nach gedreht, sobald sie die Riemen berühren. Um eine kontinuierliche Förderung zu gewährleisten, ist es sehr wichtig, dass die Korken sofort nach dem Auftreffen auf dem Kanal den Auffangsbereich wieder verlassen, damit nachfolgende Korken nicht behindert werden. Es wird deutlich, dass die Funktion der Walze, die die Korken vorvereinzelt, sehr wichtig ist. Je gleichmäßiger die Walze die Korken auf die Kanäle fördert, desto zuverlässiger funktioniert die Anlage und desto höher ist der zu realisierende Durchsatz. Gesucht wird also ein Kompromiss, um sowohl die kontinuierliche Förderung als auch die Vereinzelung der Korken sicherzustellen. Bleiben Korken auf einem Profil liegen, werden sie durch einen Impuls von einem nachkommenden Korken wieder in Bewegung gebracht. Da die Reibung zwischen Gummiriemen und Korken größer ist als die Reibung zwischen Profil und Korken, reicht die Berührung mit dem Riemen, um die Korken zu drehen und zu fördern. Liegen zwei Korken parallel übereinander, sorgt die unterschiedliche Reibung zwischen Riemen und Korken, Profil und Korken und den Korken untereinander dafür, dass der untere Korken gefördert wird, und der obere Korken, der teils auf dem Profil, teils auf dem unteren Korken liegt, sich langsamer fortbewegt und sich hinter dem auf den Riemen befindlichen Korken einreiht.

Bei der Auslegung der Vereinzelungseinrichtung spielen mehrere Faktoren eine Rolle: teilweise sind die Korken staubig, klebrig oder anderweitig verschmutzt, es liegen unterschiedliche Reibwerte vor, es ist möglich, dass sehr viel gleichartige Korken nacheinander ankommen (z. B. große Sektkorken). Sollte die Länge der Profile nicht ausreichen, um die Korken zu vereinzeln (z. B. zwei Korken werden nebeneinander gefördert), so wird am Ende des Profils (3) nur der Korken weitergefördert, der zwischen den Riemen liegt, der andere fällt neben den Riemen zwischen den Kanälen durch und muss über eine Rückführung erneut der Anlage zugeführt werden. Die Korken, die richtig auf den Riemen positioniert sind, werden der Identifikationseinheit an Position (4) zugeführt. 


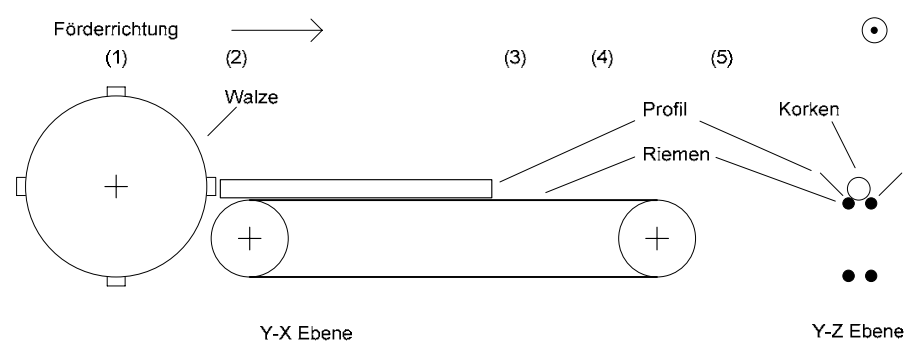

Abbildung 11: Schema der Vereinzelungs- und Fördereinrichtung

\section{Sortierkonzepte}

Auf Basis der Untersuchungsergebnisse wurden sechs Sortierkonzepte entwickelt. Als wichtigste Kriterien, ob sich eine physikalische Korkeigenschaft als Trennkriterium eignet, stellten sich die Geometrie- und Abstandsempfindlichkeit eines Messverfahrens, die Geschwindigkeit des Messverfahrens und die Störanfälligkeit heraus. Nachfolgend werden vier der Verfahren kurz beschrieben. Das im Prototyp eingesetzte Verfahren wird im letzten Teil dieser Abhandlung näher betrachtet. Bei allen Konzepten ist eine manuelle Vorsortierung zur Beseitigung von Altbatterien, Papier, ... und sonstigem Müll sowie eine Fe-Metallabscheidung vorgeschaltet.

\subsection{Konzept: Thermische Trennung}

Dieses Verfahren beruht auf den Hafteigenschaften der Kunststoffflaschenstopfen bei Temperaturen über der Erweichungstemperatur. Aufgrund der großen Unterschiede der Erweichungstemperaturen der Kunststoffflaschenstopfen wird ein mindestens zweistufiges Verfahren erforderlich (Abbildung 12).

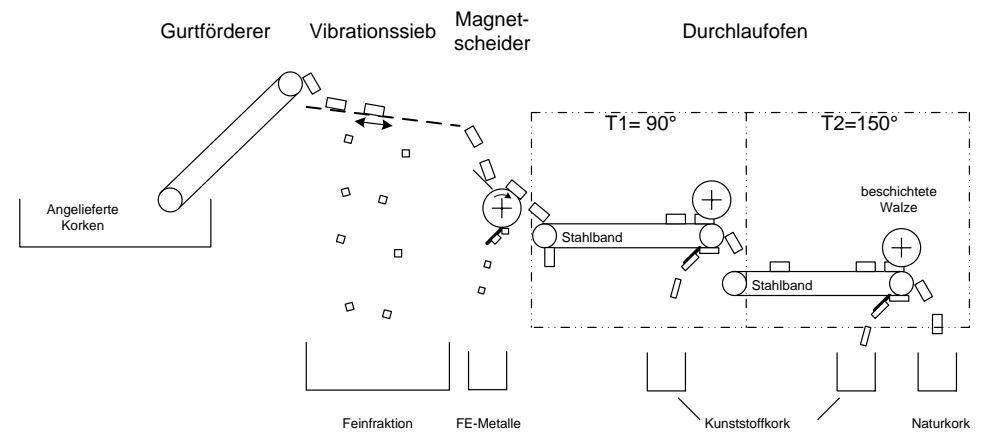

Abbildung 12: Anlagenschema Thermische Trennung (2stufiges Verfahren)

In der ersten Stufe werden die Korken in einem Durchlaufofen auf eine Temperatur von etwa $90^{\circ} \mathrm{C}$ erhitzt. Am Ende der Erwärmungsstrecke werden die Korken mit einer vorgewärmten Walze auf das Metallband gepresst, um die Haftneigung der erweichten Kunststoffe zu verstärken. Die nicht haftenden Korken werden am Bandende abgeworfen (Wurfparabel), während die erweichten Korken am Metallband haften. Sie werden mit einem Abstreifer noch im bzw. nach dem Umlenkbereich vom Band gelöst und ausgeschleust. Dieser Vorgang kann durch Abkühlung des Bandes verstärkt werden. In der zweiten Stufe findet der gleiche Vorgang statt, wobei die Korken auf eine Temperatur von ca. $150^{\circ} \mathrm{C}$ erhitzt werden.

Vorteile dieses Verfahrens sind die kleinen bis mittleren Anschaffungskosten und eine annähernd sortenreine Kunststofffraktion. Nachteilig ist ein mögliches Verkleben von Walze, Band und Naturkorken bzw. Abfällen, sowie die hohen Betriebskosten bedingt durch den hohen Energieaufwand. 


\subsection{Konzept: Spektroskopie (vorzugsweise im NIR-Bereich)}

Für dieses Verfahren werden die Unterschiede in den NIR-Spektren der Korken (und anderer Objekte) ausgenutzt. Die Korken müssen zunächst vereinzelt und auf ein oder mehrere Kanäle aufgeteilt werden (je nach Anzahl der Spektrometer). Die Korken werden mit einer Lichtquelle bestrahlt und das reflektierte Spektrum aufgenommen. Durch Vergleich der aufgenommenen Spektren mit hinterlegten Spektren in einer Auswerteeinheit (IPC) kann der Werkstoff des erfassten Korken (oder eines anderen Objekts) bestimmt werden. Die unterschiedlichen Werkstoffe können dann sortenrein ausgeschleust werden.

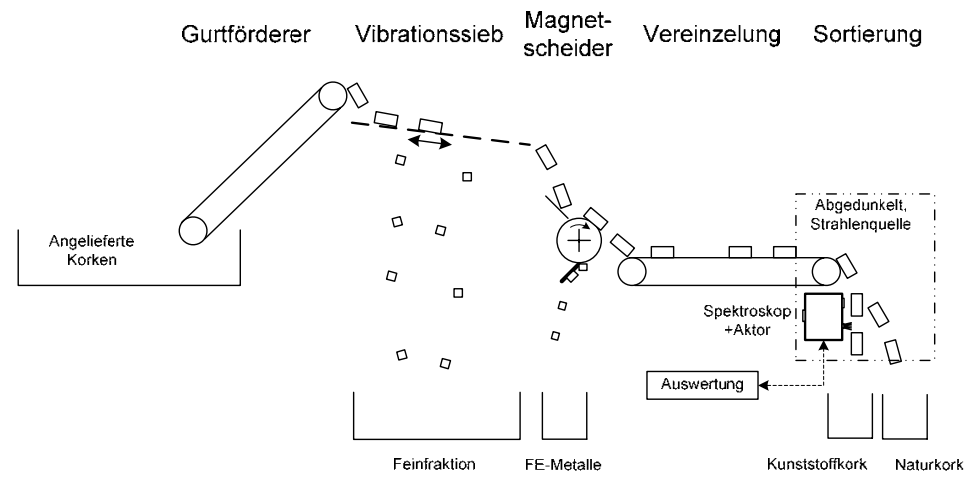

Abbildung 13: Anlagenschema Spektroskopie

Die Vorteile des Verfahrens sind die Erkennung fast aller anzutreffenden Werkstoffe, wodurch eine sortenreine Trennung möglich wird (Unterscheidung unterschiedlicher Kunststoffe) und die niedrigen Betriebskosten. Der größte Nachteile ist, dass bedingt durch die hohen Durchsatzanforderungen mehrere Spektrometer erforderlich sind. Die Anlagenkosten sind demnach sehr groß.

\subsection{Konzept: Schwarzlicht + Farbsortierung mit Bildverarbeitung}

Auch dieses Verfahren nutzt die Reflexions- und Fluoreszenzeigenschaften der Kunststoff- und Naturkorken. Die Korken werden mit Schwarzlicht bestrahlt. Die Naturkorken fluoreszieren über ein größeres Spektrum, während die Kunststoffflaschenstopfen fast ausschließlich reflektieren.

Die Korken dürfen nicht übereinander vorliegen, müssen deshalb „einfach vereinzelt“ werden. In einen abgedunkelten Bereich der Anlage werden sie mit Schwarzlicht bestrahlt und mit einer Farbzeilenkamera abgetastet. In einer Auswerteeinheit (IPC) wird Farbe und Position der Korken bestimmt. Die Korken, die einen hohen Grünanteil aufweisen (Aufteilung der Farbkanäle) sind bis auf wenige Ausnahmen Naturkorken (siehe Abbildung 7 bis Abbildung 9). Entsprechend ihrer Position werden Aktoren zum Aussondern der Naturkorken angesprochen.

Durch Erweiterung dieses Verfahrens auf Geometrieerkennung ist es möglich weitere Objekte, wie Griffkorken, auszusortieren.

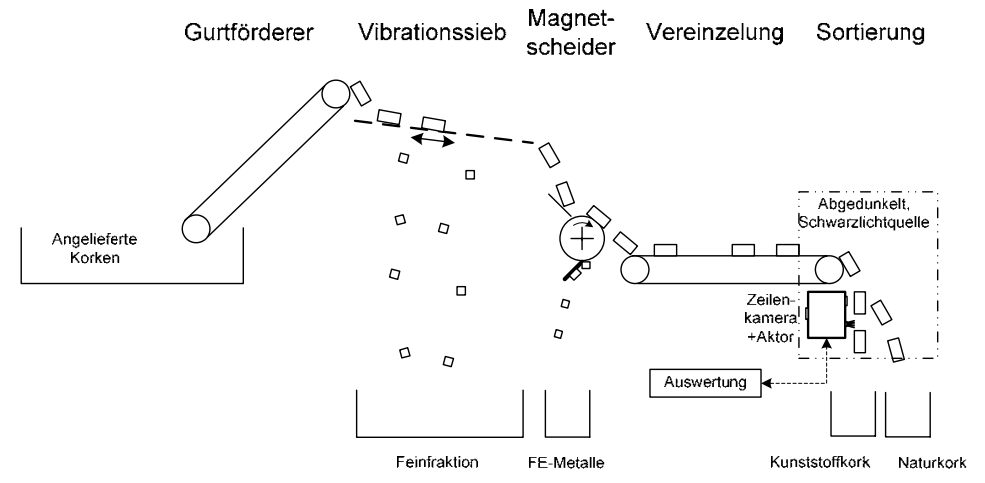

Abbildung 14: Anlagenschema Schwarzlicht + Bildverarbeitung 
Auch bei diesem Verfahren kann von geringen Betriebskosten ausgegangen werden. Sein größter Vorteil ist, die Erkennung von Form- und Größenunterschieden. In diesem Fall ist allerdings die erforderliche Rechenzeit so groß, dass auch hier mehrere Systeme erforderlich sind. Die Anschaffungskosten können somit nicht als gering eingestuft werden. Ein weiterer Nachteil ist, dass der Sensorbereich sehr sorgfältig abgedunkelt sein muss.

\subsection{Konzept: Mikrowellenbestrahlung + Temperaturmessung}

Dieses Verfahren nutzt den Wassergehalt der Naturkorken. Wie bei den Eigenschaften des Sortierguts beschrieben ist damit unter bestimmten Randbedingungen eine deutliche Unterscheidung von Natur- und Kunststoffflaschenstopfen möglich. Um das Ergebnis nicht zu verfälschen muss sichergestellt sein, dass die Korkoberflächen keine Restfeuchte von Transport und Lagerung aufweisen. Dazu durchlaufen die Korken einen klimatisierten Anlagenabschnitt (Abbildung 15) in dem ebenfalls die Temperatur der Korken auf ein gleiches vorgegebenes Niveau gebracht wird und die Korken vereinzelt werden (auf Kanäle). Die Temperatur der Korken wird gemessen und einer Auswerteeinheit zur Verfügung gestellt. Anschließend durchlaufen die Korken einen Mikrowellentunnel. Die Naturkorken werden aufgrund ihres Wassergehalts erwärmt, während die Kunststoffflaschenstopfen ihre Temperatur beibehalten. Nach der Mikrowellenbestrahlung wird je Kanal die Temperatur der Korken gemessen und in der Auswerteeinheit die Temperaturdifferenz bestimmt. Übersteigt die Temperaturdifferenz einen bestimmten Schwellwert, liegt ein Naturkork vor. Ein Aktor wird angesprochen und der Naturkork wird ausgeschleust.

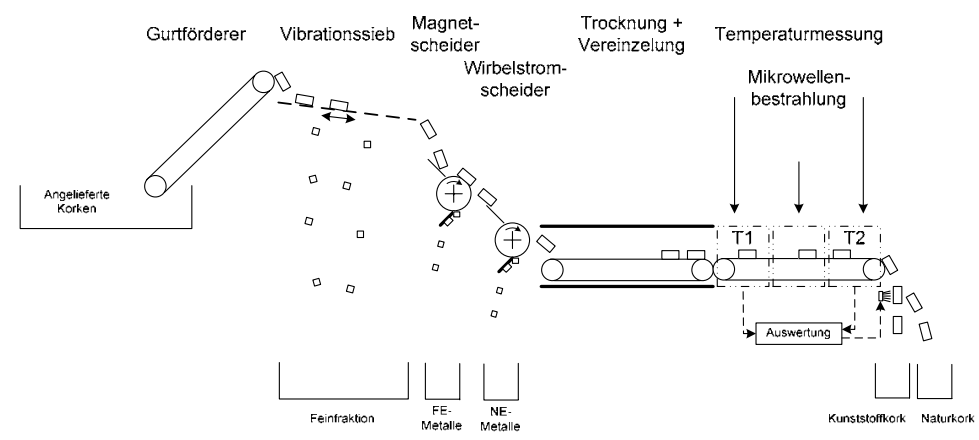

Abbildung 15: Anlagenschema Mikrowellenanregung

Dieses Verfahren bietet nach der Spektroskopie die zuverlässigste Unterscheidung von Natur- und Kunststoffflaschenstopfen. Nachteilig ist die Tatsache, dass Korken mit Naturkork- und Kunststoffbestandteilen als Naturkork detektiert werden. Als weit größerer Nachteil ist der Aufwand für das Einhalten aller Sicherheitsstandards und Arbeitssicherheitsvorschriften einzuschätzen. So ist beispielsweise eine 100\%ige Metallabscheidung erforderlich und aufgrund der Abschirmung ist ein Durchlaufofen nur schwer realisierbar. Mit dem erforderlichen Energieaufwand sind außerdem die Betriebskosten als sehr hoch einzuschätzen.

Mit weit weniger Energie kommt die Mikrowellen-Feuchtemessung aus. Dieses Verfahren ist allerdings abstands- und geometrieabhängig und nur zum Teil für die Feuchtemessung von Feststoffen geeignet.

\subsection{Zur Umsetzung ausgewähltes Verfahren (Prototyp)}

Für die technische Umsetzung wurden die entwickelten Sortierkonzepte nach ihrer Prozesssicherheit, dem konstruktiven Aufwand, der Möglichkeit der Durchsatzerhöhung, der Wartungsfreundlichkeit, der sensortechnischen Umsetzungsmöglichkeit und der Wirtschaftlichkeit bewertet. Ausgewählt wurde ein Konzept, das auf der Ausnutzung unterschiedlicher optischer Eigenschaften von Natur- und Kunststoffflaschenstopfen beruht.

Um den geforderten Durchsatz zu erreichen, werden die Korken im Prototyp mit der oben beschriebenen Vereinzelungseinrichtung auf 12 Kanäle vereinzelt. Jeder Kanal besitzt eine Sensor-Aktor-Einheit (Abbildung 16) mit zwei neu entwickelten LowCost-Sensoren, die in unterschiedlichen Wellenlängen arbeiten, einer analogen Signalvorverarbeitung der beiden Sensorsignale (Abbildung 20) und einem Aktor, der aus einem Magnetventil und einer Druckluftdüse besteht. Die digitale Signalverarbeitung, die Vorgabe der Vergleichswerte (siehe Abbildung 20) und die Ansteuerung des Aktors erfolgt in einer zentralen Steuerung für alle 12 Kanäle. Die Sensor-Aktor-Einheiten sind einzeln austauschbar, wodurch eine hohe Verfügbarkeit und die Möglichkeit durch Hinzufügen von Kanälen den Gesamtdurchsatz der Anlage bei Bedarf zu erhöhen, gewährleistet wird. 
Die eigentliche Sortierung bzw. Verzweigung in einen „Gutkork-Strom“ und einen „Kunststoffkork-Strom“ erfolgt durch Aussondern der erkannten Kunststoffflaschenstopfen. Die Kunststoffflaschenstopfen werden dabei durch einen Druckluftimpuls quer zur Förderrichtung von den Transportriemen gestoßen, während Natur-, Pressund Feingranulatkorken auf den Transportriemen verbleiben. Ein geringer Anteil Naturkork, der aufgrund von Störeinflüssen und Überschneidungsbereichen der physikalischen Eigenschaften mit aussortiert werden muss, wird an einem Sortierband manuell erfasst und den Gutkorken wieder zugeführt. Mit dieser Positivauswahl bei der Prüfung eines sehr geringen Teilstroms wird die Qualität des Endprodukts sichergestellt.
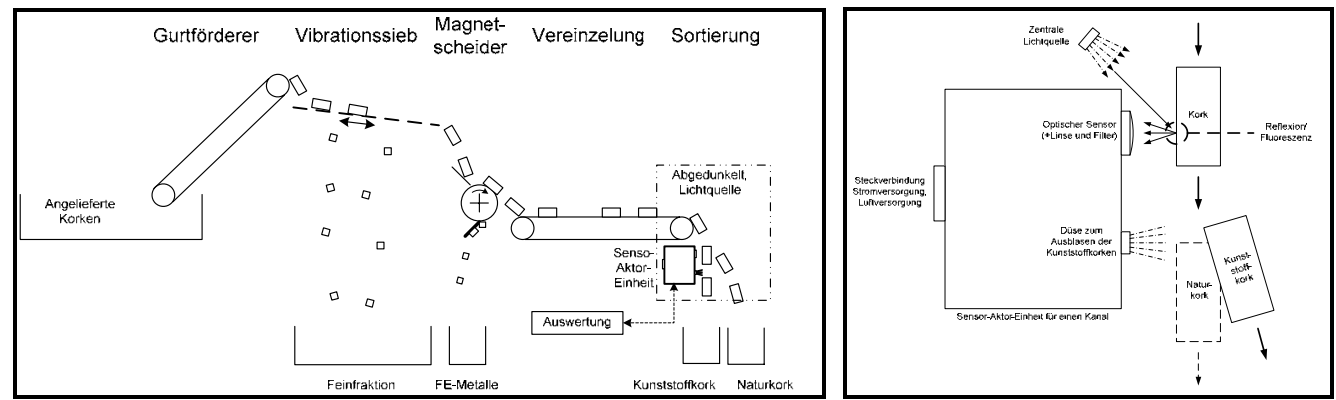

Abbildung 16: Anlagenschema des Prototyps und Schema der Sensor-Aktor-Einheit

Die drei nachfolgenden Abbildungen zeigen den Verlauf der verstärkten und vorverarbeiteten Sensorsignalen beim Passieren der Sensor-Aktor-Einheit durch einen Korken. Die Schwellen, die das Ansprechen des binären Lichtschrankensignals (im Diagramm: „Lichtschranke“) bedingen, sind nicht abgebildet.

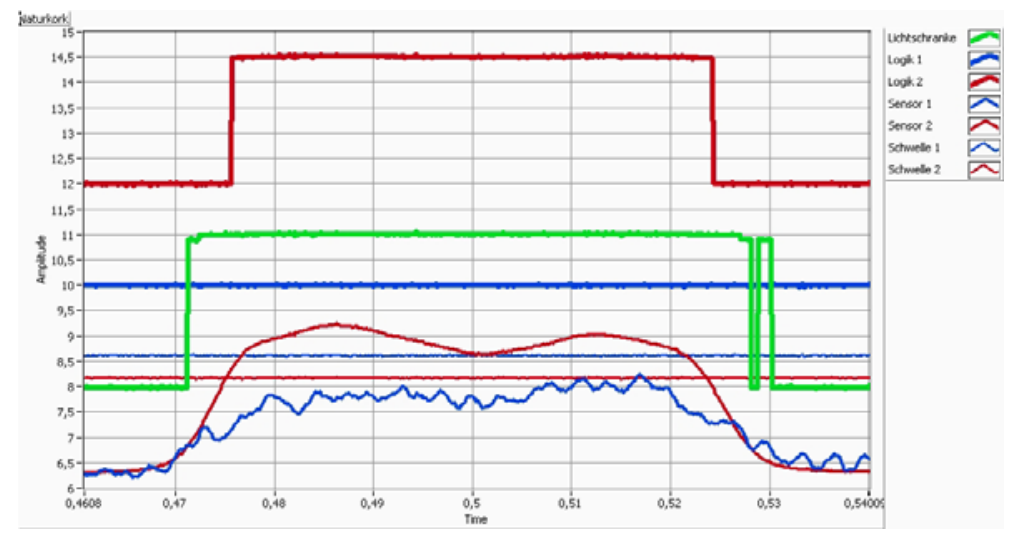

Abbildung 17: Erfassung eines Naturkorken

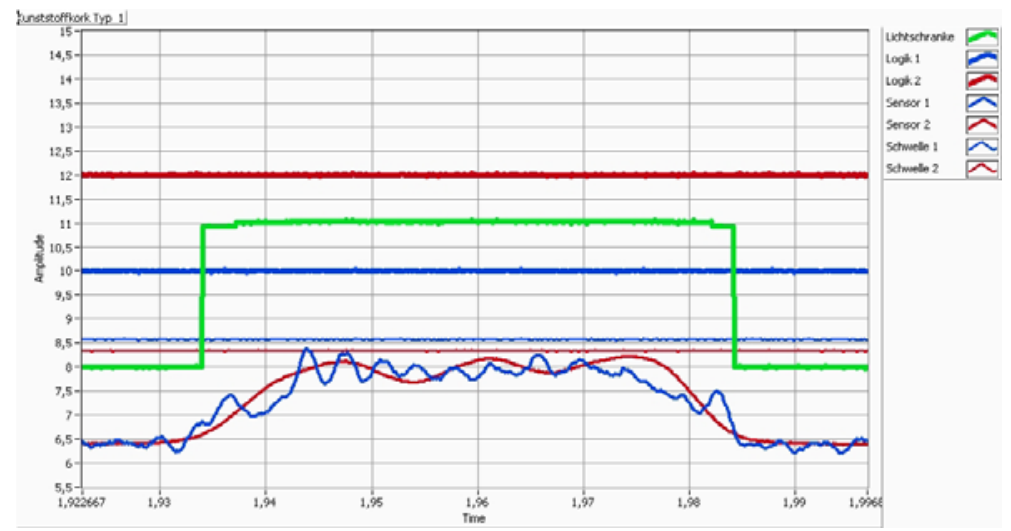

Abbildung 18: Erfassung eines Kunststoffflaschenstopfens vom Typ 1 


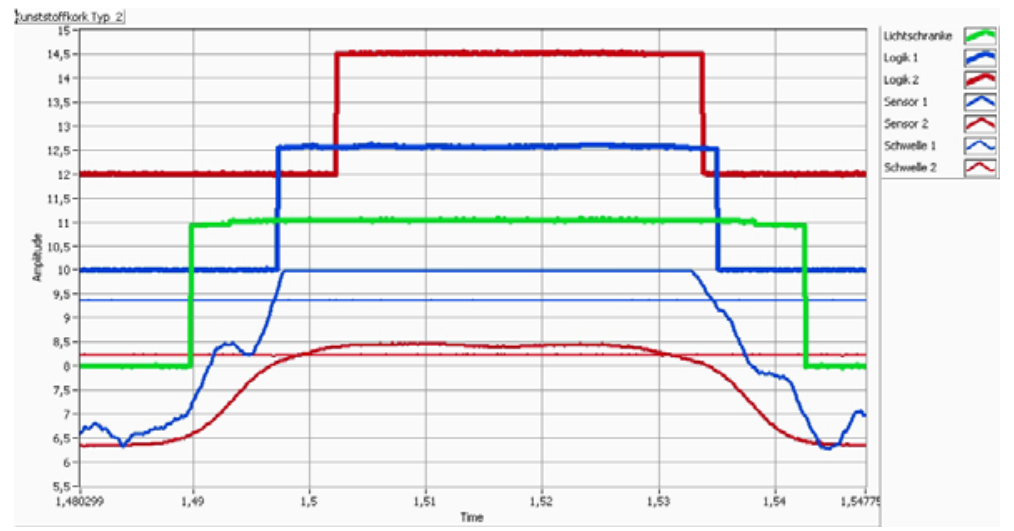

Abbildung 19: Erfassung eines Kunststoffflaschenstopfens vom Typ 2

Die Verarbeitung der Signale beinhaltet neben den in Abbildung 20 gezeigten Gliedern noch zwei Zeitglieder, die das Ein- und Ausfahren des Korks in den bzw. aus dem Sensorbereich berücksichtigen. Überschreitet der analoge Signalwert eines Sensors („Sensor 1“ und „Sensor 2“) den zugehörigen Schwellwert (im Diagram: „Schwelle 1“ und „Schwelle 2“), wird das entsprechende binäre Logiksignal („Logik 1“ und „Logik 2“) gesetzt und beim Unterschreiten der Schwelle wieder gelöscht. Für die Erkennung eines Natur-/Press-/Feingranulatkorks muss das Lichtschrankensignal und das zu Sensor 2 gehörende Logiksignal („Logik 2“) gesetzt sein. Die unterschiedlichen Kunststoffflaschenstopfen können für die Erkennung in zwei Typen unterteilt werden. Typ 1 ist gekennzeichnet durch zwei sehr niedrige Sensorsignale und löst den Aktor bei gesetzter Lichtschranke und gelöschten Logiksignalen („Logik 1“, „Logik 2“) aus. Ein Kunststoffkork vom Typ 2 liegt vor, wenn die Lichtschranke und das zu Sensor 1 gehörende Logiksignal („Logik 1“) gesetzt sind. Der Zustand von „Logik 2“ spielt dabei keine Rolle.

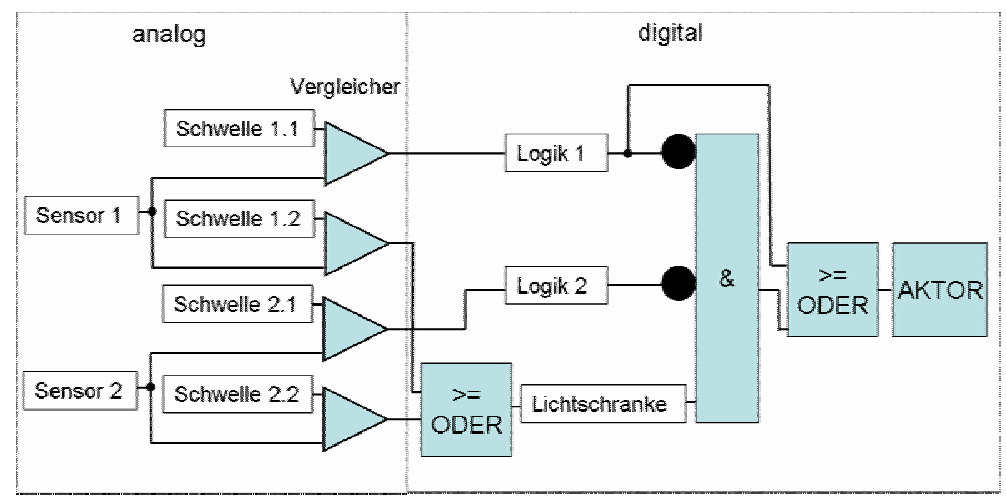

Abbildung 20: Verarbeitung der zwei Sensorsignale

Mit dem Prototyp KORDULA (KORK DURCH LICHT AUSSORTIERT), der sich seit Januar 2006 im täglichen Einsatz befindet (Abbildung 21), wird eine Qualitätssteigerung des Recyclingkorks sowie eine Erhöhung des Durchsatzes (bis $>40$ Korken/s) bei hohem Kunststoffkorkanteil erreicht. Durch die Reduzierung des auszusortierenden Anteils um die Kunststoffflaschenstopfen in der manuellen Vorsortierung und den sehr geringen Teilstrom bei der Nachsortierung wird weiterhin eine Verbesserung der Arbeitsbedingungen des Sortierpersonals erreicht. Derzeit werden über 50 unterschiedliche Kunststoffflaschenstopfen erkannt und aussortiert. 


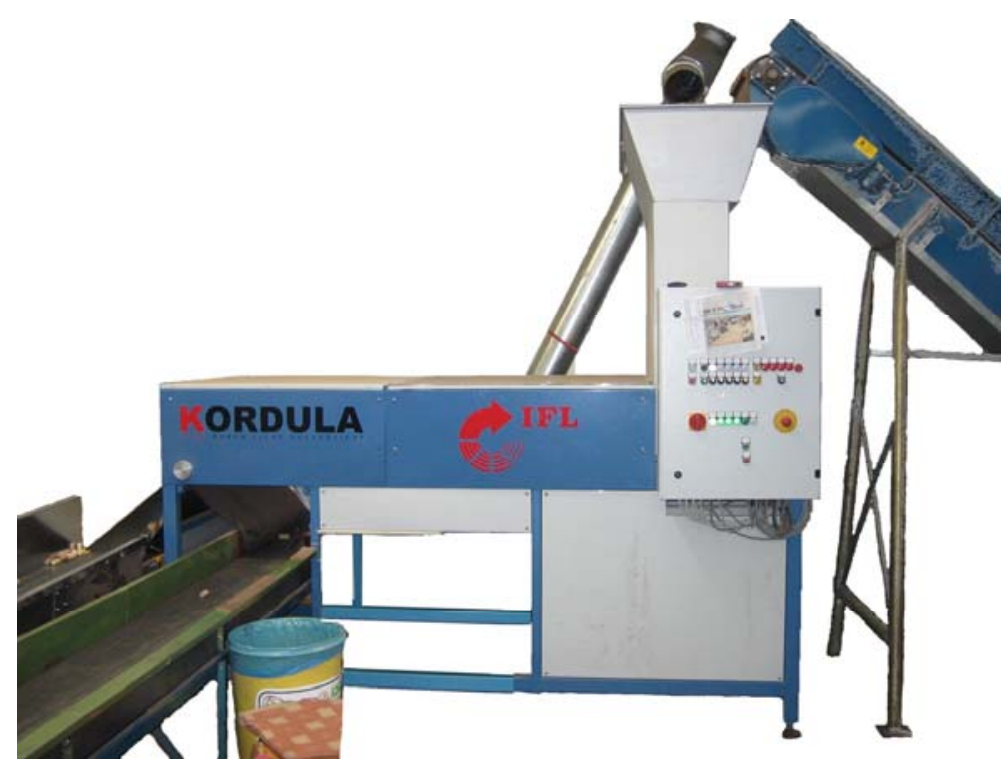

Abbildung 21: Der Prototyp im täglichen Einsatz

\section{Literatur}

[Thom83]

[VDI93]

[VDI98]
Karl J. Thomé-Kozmiensky (Hrsg.), Materialrecycling durch Abfallaufbereitung, E: Freitag Verlag für Umweltttechnik, Berlin, 1983

Aufbereitungstechnik beim Verwerten von Kunststoffen, Herausgeber: Verein Deutscher Ingenieure, VDI-Gesellschaft Kunststofftechnik, VDI Verlag, Düsseldorf, 1993

Dipl.-Chem. Dipl-Wirtsch.-Chem Ingo Burmester, Lasergestützte

Kunststofferkennung im Recyclingprozess, Fortschrittberichte VDI Reihe 5: Grundund Werkstoffe Nr. 517, Hannover, VDI Verlag GmbH, 1998 